\title{
Correction to: Systematic Review of Conceptual, Age, Measurement and Valuation Considerations for Generic Multidimensional Childhood Patient-Reported Outcome Measures
}

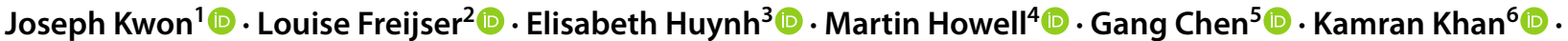

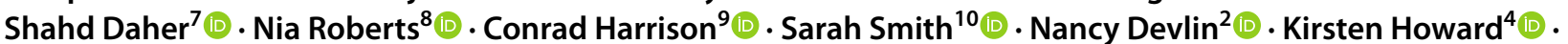

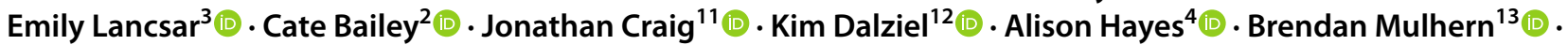 \\ Germaine Wong ${ }^{4}\left(\mathbb{C} \cdot\right.$ Julie Ratcliffe ${ }^{14}(\mathbb{C}) \cdot$ Stavros Petrou ${ }^{7}(\mathbb{D}$
}

Published online: 14 February 2022

(c) The Author(s) 2022

\section{Correction to: PharmacoEconomics} https://doi.org/10.1007/s40273-021-01128-0

In the original version of this article, the author's name Emily Lancsar was incorrectly written as Emily Lanscar.

The original article has been corrected.

Open Access This article is licensed under a Creative Commons Attribution-NonCommercial 4.0 International License, which permits any non-commercial use, sharing, adaptation, distribution and reproduction

The original article can be found online at https://doi.org/10.1007/ s40273-021-01128-0.

Stavros Petrou

stavros.petrou@phc.ox.ac.uk

Joseph Kwon

jkwon6@sheffield.ac.uk

Louise Freijser

louise.freijser@unimelb.edu.au

Elisabeth Huynh

elisabeth.huynh@anu.edu.au

Martin Howell

martin.howell@sydney.edu.au

Gang Chen

gang.chen@monash.edu

Kamran Khan

k.a.khan@warwick.ac.uk

Shahd Daher

shahd.daher@phc.ox.ac.uk

Nia Roberts

nia.roberts@bodleian.ox.ac.uk

Conrad Harrison

conrad.harrison@balliol.ox.ac.uk in any medium or format, as long as you give appropriate credit to the original author(s) and the source, provide a link to the Creative Commons licence, and indicate if changes were made. The images or other third party material in this article are included in the article's Creative Commons licence, unless indicated otherwise in a credit line to the material. If material is not included in the article's Creative Commons licence and your intended use is not permitted by statutory regulation or exceeds the permitted use, you will need to obtain permission directly from the copyright holder. To view a copy of this licence, visit http://creativecommons.org/licenses/by-nc/4.0/. 
1 School of Health and Related Research, University of Sheffield, Sheffield, England, UK

2 Centre for Health Policy, University of Melbourne, Melbourne, Australia

3 Department of Health Services Research and Policy, Australian National University, Canberra, Australia

4 School of Public Health, University of Sydney, Sydney, Australia

5 Centre for Health Economics, Monash University, Melbourne, Australia

6 Centre for Health Economics at Warwick, University of Warwick, Coventry, England, UK

7 Nuffield Department of Primary Care Health Sciences, University of Oxford, Oxford, England, UK

8 Bodleian Health Care Libraries, University of Oxford, Oxford, England, UK
$9 \quad$ Nuffield Department of Orthopaedics, Rheumatology and Musculoskeletal Sciences, University of Oxford, Oxford, England, UK

10 Department of Health Services Research and Policy, London School of Hygiene and Tropical Medicine, London, England, UK

11 College of Medicine and Public Health, Flinders University, Adelaide, Australia

12 Health Economics Unit, University of Melbourne, Melbourne, Australia

13 Centre for Health Economics Research and Evaluation, University of Technology Sydney, Sydney, Australia

14 Caring, Futures Institute, College of Nursing and Health Sciences, Flinders University, Adelaide, Australia 